ANNALES

POLONICI MATHEMATICI

LXXVII.1 (2001)

\title{
On distance between zeros of solutions of third order differential equations
}

\author{
by N. Parhi and S. Panigrahi (Berhampur)
}

\begin{abstract}
The lower bounds of the spacings $b-a$ or $a^{\prime}-a$ of two consecutive zeros or three consecutive zeros of solutions of third order differential equations of the form

$$
y^{\prime \prime \prime}+q(t) y^{\prime}+p(t) y=0
$$

are derived under very general assumptions on $p$ and $q$. These results are then used to show that $t_{n+1}-t_{n} \rightarrow \infty$ or $t_{n+2}-t_{n} \rightarrow \infty$ as $n \rightarrow \infty$ under suitable assumptions on $p$ and $q$, where $\left\langle t_{n}\right\rangle$ is a sequence of zeros of an oscillatory solution of $(*)$. The Opial-type inequalities are used to derive lower bounds of the spacings $d-a$ or $b-d$ for a solution $y(t)$ of $(*)$ with $y(a)=0=y^{\prime}(a), y^{\prime}(c)=0$ and $y^{\prime \prime}(d)=0$ where $d \in(a, c)$ or $y^{\prime}(c)=0$, $y(b)=0=y^{\prime}(b)$ and $y^{\prime \prime}(d)=0$ where $d \in(c, b)$.
\end{abstract}

1. It is well known (see [9]) that if $y(t)$ is a solution of

$$
y^{\prime \prime}+p(t) y=0
$$

with $y(a)=0=y(b)(a<b)$ and $y(t) \neq 0$ for $t \in(a, b)$, then

$$
(b-a) \int_{a}^{b}|p(t)| d t>4 \text {. }
$$

In [8], Hartman obtained an inequality which is more general than (2). The inequality (2) is used to study the disconjugacy of (1) on an interval. It was generalized to second order nonlinear differential equations by Eliason [4], to delay-differential equations of second order by Eliason [5, 6] and Dahiya and Singh [2] and to higher order differential equations by Pachpatte [11]. However, the results in [11] are not applicable to odd order differential equations. In a recent work [13], the authors have obtained Lyapunov-type inequality for third order differential equations of the form

$$
y^{\prime \prime \prime}+p(t) y=0 \text {. }
$$

2000 Mathematics Subject Classification: 34C10, 34C15.

Key words and phrases: zeros of a solution, lower bounds of zeros, third order differential equations. 
This inequality is used to study many interesting properties of the zeros of an oscillatory solution of (3) (see Theorems 5 and 6 in [13]). In particular, the following result is obtained :

Theorem A. Let $p \in L^{\sigma}([0, \infty), \mathbb{R})$, where $1<\sigma<\infty$. If $\left\langle t_{n}\right\rangle$ is an increasing sequence of zeros of an oscillatory solution $y(t)$ of (3), then $t_{n+1}-t_{n} \rightarrow \infty$ or $t_{n+2}-t_{n} \rightarrow \infty$ as $n \rightarrow \infty$.

A result similar to Theorem A concerning (1) was obtained by Patula [14]. In [16], Yan extended the result of Patula to hold for $0<\sigma<\infty$. He obtained the following result:

TheOREM B. Let $0<\sigma<\infty$. Suppose that $p(t)$ is a real-valued function on $[0, \infty)$ with $|p(t)|^{\sigma}$ locally integrable and, for a constant $\delta_{0}>0$,

$$
\lim _{t \rightarrow \infty} \int_{t}^{t+\delta_{0}}|p(s)|^{\sigma} d s=0 .
$$

If $y(t)$ is any oscillatory solution of (1), then the distance between consecutive zeros of $y(t)$ must become infinite.

In a recent paper [1], Brown and Hinton used Opial's inequality to obtain lower bounds for the spacing of zeros of a solution of (1) and to obtain lower bounds for the spacing $\beta-\alpha$ where $y(t)$ is a solution of (1) satisfying $y(\alpha)=y^{\prime}(\beta)=0$ or $y^{\prime}(\alpha)=y(\beta)=0(\alpha<\beta)$.

The purpose of this work is (i) to extend the results in [13] to third order differential equations of the form

$$
y^{\prime \prime \prime}+q(t) y^{\prime}+p(t) y=0,
$$

where $p$ and $q$ are real-valued functions on $[0, \infty)$ such that $q$ is once differentiable and each of $p(t)$ and $q^{\prime}(t)$ is locally integrable, (ii) to obtain a result similar to Theorem A under weaker assumption on $p$ and for $0<\sigma<\infty$ and (iii) to obtain lower bounds for the spacing $d-a$ with the help of Opial-type inequalities, where $y(t)$ is a solution of (4) with $y(a)=y^{\prime}(a)=0, y^{\prime}(c)=0$ and $y^{\prime \prime}(d)=0(a<d<c)$. We consider the problems (i) and (ii) in Section 2 and the problem (iii) in Section 3.

2. We need the following lemma (see [16]).

Lemma 1. Let $f$ be a locally integrable nonnegative function on $[0, \infty)$. If

$$
\lim _{t \rightarrow \infty} \int_{t}^{t+\delta_{0}} f(s) d s=0
$$


for some $\delta_{0}>0$, then

$$
\lim _{t \rightarrow \infty} \int_{t}^{t+\delta} f(s) d s=0
$$

for every $\delta>0$.

Let $y(t)$ be a solution of (4) with $y(a)=y(b)=0(0 \leq a<b)$ and $y(t) \neq 0$ for $t \in(a, b)$. We consider the following two cases:

CASE I: $y^{\prime \prime}(d)=0$ for some $d \in[a, b]$.

CASE II: $y^{\prime \prime}(t) \neq 0$ for $t \in[a, b]$. In this case we consider three consecutive zeros of $y(t)$, viz., $y(a)=0, y(b)=0, y\left(a^{\prime}\right)=0\left(0 \leq a<b<a^{\prime}\right)$, $y(t) \neq 0$ for $t \in(a, b)$ and $y(t) \neq 0$ for $t \in\left(b, a^{\prime}\right)$.

Theorem 2. Consider Case I. Then

$$
(b-a)\left[\int_{a}^{b}|q(t)| d t+(b-a)|q(d)|+(b-a) \int_{a}^{b}\left|q^{\prime}(t)-p(t)\right| d t\right] \geq 4 .
$$

Proof. Let $M=\max \{|y(t)|: t \in[a, b]\}=|y(c)|$, where $c \in(a, b)$. Hence

$$
M=|y(c)|=\left|\int_{a}^{c} y^{\prime}(t) d t\right| \leq \int_{a}^{c}\left|y^{\prime}(t)\right| d t
$$

and

$$
M=|y(c)|=\left|\int_{c}^{b} y^{\prime}(t) d t\right| \leq \int_{c}^{b}\left|y^{\prime}(t)\right| d t
$$

Thus

$$
2 M \leq \int_{a}^{b}\left|y^{\prime}(t)\right| d t
$$

Applying the Cauchy-Schwarz inequality first and then integrating by parts, we obtain

$$
\begin{aligned}
4 M^{2} & \leq\left[\int_{a}^{b}\left|y^{\prime}(t)\right| d t\right]^{2} \leq(b-a) \int_{a}^{b}\left(y^{\prime}(t)\right)^{2} d t \\
& =-(b-a) \int_{a}^{b} y(t) y^{\prime \prime}(t) d t \leq(b-a) \int_{a}^{b}|y(t)| \cdot\left|y^{\prime \prime}(t)\right| d t .
\end{aligned}
$$

Integrating (4) from $d$ to $t(a \leq d<t$ or $t<d \leq b)$ we get

$$
\begin{aligned}
y^{\prime \prime}(t) & =-\int_{d}^{t} q(s) y^{\prime}(s) d s-\int_{d}^{t} p(s) y(s) d s \\
& =-q(t) y(t)+q(d) y(d)+\int_{d}^{t}\left(q^{\prime}(s)-p(s)\right) y(s) d s,
\end{aligned}
$$


that is,

$$
\begin{aligned}
\left|y^{\prime \prime}(t)\right| & \leq M\left[|q(t)|+|q(d)|+\left|\int_{d}^{t}\right| q^{\prime}(s)-p(s)|d s|\right] \\
& \leq M\left[|q(t)|+|q(d)|+\int_{a}^{b}\left|q^{\prime}(t)-p(t)\right| d t\right] .
\end{aligned}
$$

Hence

$$
4 M^{2} \leq(b-a) M^{2}\left[\int_{a}^{b}|q(t)| d t+(b-a)|q(d)|+(b-a) \int_{a}^{b}\left|q^{\prime}(t)-p(t)\right| d t\right]
$$

that is,

$$
4 \leq(b-a)\left[\int_{a}^{b}|q(t)| d t+(b-a)|q(d)|+(b-a) \int_{a}^{b}\left|q^{\prime}(t)-p(t)\right| d t\right] .
$$

Thus the theorem is proved.

Theorem 3. Consider Case II. Then

$$
\left(a^{\prime}-a\right)\left[\int_{a}^{a^{\prime}}|q(t)| d t+\left(a^{\prime}-a\right)|q(d)|+\left(a^{\prime}-a\right) \int_{a}^{a^{\prime}}\left|q^{\prime}(t)-p(t)\right| d t\right] \geq 4 .
$$

Proof. There exist $c_{1} \in(a, b)$ and $c_{2} \in\left(b, a^{\prime}\right)$ such that $y^{\prime}\left(c_{1}\right)=0$ and $y^{\prime}\left(c_{2}\right)=0$. Hence there exists a $d \in\left(c_{1}, c_{2}\right)$ such that $y^{\prime \prime}(d)=0$. Setting $M=\max \left\{|y(t)|: t \in\left[a, a^{\prime}\right]\right\}=|y(c)|$, where $c \in(a, b) \cup\left(b, a^{\prime}\right)$, we get

$$
M=|y(c)|=\left|\int_{a}^{c} y^{\prime}(t) d t\right| \leq \int_{a}^{c}\left|y^{\prime}(t)\right| d t
$$

and

$$
M=|y(c)|=\left|\int_{c}^{a^{\prime}} y^{\prime}(t) d t\right| \leq \int_{c}^{a^{\prime}}\left|y^{\prime}(t)\right| d t
$$

Hence $2 M \leq \int_{a}^{a^{\prime}}\left|y^{\prime}(t)\right| d t$. Then proceeding as in the proof of Theorem 2 we obtain the required inequality. Thus the proof of the theorem is complete.

Remark. In Case II, $d \in\left(b, a^{\prime}\right]$ such that $y^{\prime \prime}(d)=0$. By Theorem 2,

$$
\left(a^{\prime}-b\right)\left[\int_{b}^{a^{\prime}}|q(t)| d t+\left(a^{\prime}-b\right)|q(d)|+\left(a^{\prime}-b\right) \int_{b}^{a^{\prime}}\left|q^{\prime}(t)-p(t)\right| d t\right] \geq 4
$$

However, one cannot get the lower bound of the spacing $b-a$ from (6) and (7). 
REMARK. If $q(t) \equiv 0, t \in[0, \infty)$, then (5) and (6) reduce, respectively, to

$$
(b-a)^{2} \int_{a}^{b}|p(t)| d t \geq 4
$$

and

$$
\left(a^{\prime}-a\right)^{2} \int_{a}^{a^{\prime}}|p(t)| d t \geq 4 .
$$

These inequalities were obtained in [13].

REMARK. If $y(t)$ is a solution of (4) with $y(a)=0=y^{\prime}(a), y(b)=0$ and $y(t) \neq 0$ for $t \in(a, b)$, then there exists a $d \in(a, b)$ such that $y^{\prime \prime}(d)=0$ and hence (5) holds. Similarly, if $y(t)$ is a solution of (4) with $y(a)=0$, $y(b)=0=y^{\prime}(b)$ and $y(t) \neq 0$ for $t \in(a, b)$, then there exists a $d \in(a, b)$ such that $y^{\prime \prime}(d)=0$ and hence (5) holds.

REMARK. In many cases it is not easy to find $d$ explicitly in $(a, b)$ such that $y^{\prime \prime}(d)=0$. Hence the following corollaries will be useful.

Corollary 4. Consider Case I. If $q(t) \geq 0$ and $q^{\prime}(t) \leq 0, t \in[0, \infty)$, then

$$
(b-a)^{2}\left[2 q(a)+\int_{a}^{b}\left|q^{\prime}(t)-p(t)\right| d t\right] \geq 4 .
$$

This follows from the inequality (5) of Theorem 2.

Corollary 5. Consider Case II. If $q(t) \geq 0$ and $q^{\prime}(t) \leq 0, t \in[0, \infty)$, then

$$
\left(a^{\prime}-a\right)^{2}\left[2 q(a)+\int_{a}^{a^{\prime}}\left|q^{\prime}(t)-p(t)\right| d t\right] \geq 4 .
$$

This follows from the inequality (6) of Theorem 3.

Theorem 6. Let $q(t) \geq 0, q^{\prime}(t) \leq 0$ for $t \in[0, \infty)$ and $q(t) \rightarrow 0$ as $t \rightarrow \infty$. Suppose that

$$
\limsup _{t \rightarrow \infty}\left[\delta^{2} \int_{t}^{t+\delta}\left|q^{\prime}(s)-p(s)\right| d s\right]<4
$$

for every $\delta>0$. If $\left\langle t_{n}\right\rangle$ is an increasing sequence of zeros of an oscillatory solution $y(t)$ of (4), then $t_{n+1}-t_{n} \rightarrow \infty$ or $t_{n+2}-t_{n} \rightarrow \infty$ as $n \rightarrow \infty$.

Proof. Suppose that $y^{\prime \prime}\left(d_{n}\right)=0$ for some $d_{n} \in\left[t_{n}, t_{n+1}\right]$ for all large $n$. We claim that, in this case, $t_{n+1}-t_{n} \rightarrow \infty$ as $n \rightarrow \infty$. Otherwise, there exists a subsequence $\left\langle t_{n_{k}}\right\rangle$ of $\left\langle t_{n}\right\rangle$ such that $t_{n_{k}} \rightarrow \infty$ as $k \rightarrow \infty$ and $0<$ 
$t_{n_{k+1}}-t_{n_{k}} \leq L$ for every $k$, where $L>0$ is a constant. From Corollary 4 , we obtain

$$
\left(t_{n_{k+1}}-t_{n_{k}}\right)^{2}\left[2 q\left(t_{n_{k}}\right)+\int_{t_{n_{k}}}^{t_{n_{k+1}}}\left|q^{\prime}(t)-p(t)\right| d t\right] \geq 4
$$

that is,

$$
L^{2} \int_{t_{n_{k}}}^{t_{n_{k}}+L}\left|q^{\prime}(t)-p(t)\right| d t \geq 4-2 L^{2} q\left(t_{n_{k}}\right) .
$$

Hence

$$
L^{2} \limsup _{k \rightarrow \infty} \int_{t_{n_{k}}}^{t_{n_{k}}+L}\left|q^{\prime}(t)-p(t)\right| d t \geq 4,
$$

a contradiction to the given hypothesis.

Next suppose that for every integer $N>0$ there exists an integer $n \geq$ $N$ such that $y^{\prime \prime}(t) \neq 0$ for $t \in\left[t_{n}, t_{n+1}\right]$. In this case, we consider three consecutive zeros $t_{n}<t_{n+1}<t_{n+2}$ and show that $t_{n+2}-t_{n} \rightarrow \infty$ as $n \rightarrow \infty$. On the contrary, assume that there exists a subsequence $\left\langle t_{n_{k}}\right\rangle$ of $\left\langle t_{n}\right\rangle$ such that $t_{n_{k}} \rightarrow \infty$ as $k \rightarrow \infty$ and $0<t_{n_{k+2}}-t_{n_{k}} \leq \lambda$ for every $k$, where $\lambda>0$ is a constant. From Corollary 5 we obtain

$$
\left(t_{n_{k+2}}-t_{n_{k}}\right)^{2}\left[2 q\left(t_{n_{k}}\right)+\int_{t_{n_{k}}}^{t_{n_{k+2}}}\left|q^{\prime}(t)-p(t)\right| d t\right] \geq 4
$$

Hence

$$
\lambda^{2} \limsup _{k \rightarrow \infty} \int_{t_{n_{k}}}^{t_{n_{k}}+\lambda}\left|q^{\prime}(t)-p(t)\right| d t \geq 4
$$

a contradiction. Thus the proof of the theorem is complete.

THEOREM 7. Suppose that $q(t) \geq 0, q^{\prime}(t) \leq 0$ for $t \in[0, \infty)$ and $q(t) \rightarrow 0$ as $t \rightarrow \infty$. Let

$$
\lim _{t \rightarrow \infty} \int_{t}^{t+\delta_{0}}\left|q^{\prime}(s)-p(s)\right| d s=0
$$

for some $\delta_{0}>0$. If $\left\langle t_{n}\right\rangle$ is an increasing sequence of zeros of an oscillatory solution $y(t)$ of $(4)$, then $t_{n+1}-t_{n} \rightarrow \infty$ or $t_{n+2}-t_{n} \rightarrow \infty$ as $n \rightarrow \infty$.

This follows from Lemma 1 and Theorem 6 .

TheOREM 8. Let $0<\sigma<\infty$. Let $q(t) \geq 0, q^{\prime}(t) \leq 0$ for $t \in[0, \infty)$ and $q(t) \rightarrow 0$ as $t \rightarrow \infty$. Suppose that $\left|q^{\prime}(t)-p(t)\right|^{\sigma}$ is locally integrable on $[0, \infty)$ 
and

$$
\lim _{t \rightarrow \infty} \int_{t}^{t+\delta_{0}}\left|q^{\prime}(s)-p(s)\right|^{\sigma} d s=0
$$

for some $\delta_{0}>0$. If $\left\langle t_{n}\right\rangle$ is an increasing sequence of zeros of an oscillatory solution $y(t)$ of $(4)$, then $t_{n+1}-t_{n} \rightarrow \infty$ or $t_{n+2}-t_{n} \rightarrow \infty$ as $n \rightarrow \infty$.

Proof. The proof is divided into two parts: (i) $\sigma>1$ and (ii) $0<\sigma \leq 1$.

(i) Let $\sigma>1$. From Lemma 1 it follows that

$$
\lim _{t \rightarrow \infty} \int_{t}^{t+\delta}\left|q^{\prime}(s)-p(s)\right|^{\sigma} d s=0
$$

for every $\delta>0$. Suppose that $y^{\prime \prime}\left(d_{n}\right)=0$ for some $d_{n} \in\left[t_{n}, t_{n+1}\right]$ for every large $n$. If possible, suppose there exists a subsequence $\left\langle t_{n_{k}}\right\rangle$ of $\left\langle t_{n}\right\rangle$ such that $t_{n_{k}} \rightarrow \infty$ as $k \rightarrow \infty$ and $0<t_{n_{k+1}}-t_{n_{k}} \leq \lambda$ for every $k$, where $\lambda>0$ is a constant. From Corollary 4 we obtain

$$
\left(t_{n_{k+1}}-t_{n_{k}}\right)^{2}\left[2 q\left(t_{n_{k}}\right)+\int_{t_{n_{k}}}^{t_{n_{k+1}}}\left|q^{\prime}(t)-p(t)\right| d t\right] \geq 4
$$

Hence, by Hölder's inequality,

$$
\begin{aligned}
4-2 \lambda^{2} q\left(t_{n_{k}}\right) & \leq \lambda^{2} \int_{t_{n_{k}}}^{t_{n_{k+1}}}\left|q^{\prime}(t)-p(t)\right| d t \\
& \leq \lambda^{2}\left(t_{n_{k+1}}-t_{n_{k}}\right)^{1 / \mu}\left[\int_{t_{n_{k}}}^{t_{n_{k+1}}}\left|q^{\prime}(t)-p(t)\right|^{\sigma} d t\right]^{1 / \sigma} \\
& \leq \lambda^{2+1 / \mu}\left[\int_{t_{n_{k}}}^{t_{n_{k}}+\lambda}\left|q^{\prime}(t)-p(t)\right|^{\sigma} d t\right]^{1 / \sigma}
\end{aligned}
$$

where $1 / \sigma+1 / \mu=1$. Letting $k \rightarrow \infty$ and using (13) we obtain a contradiction. Next suppose that for every integer $N>0$ there exists an integer $n \geq N$ such that $y^{\prime \prime}(t) \neq 0$ for $t \in\left[t_{n}, t_{n+1}\right]$. Considering three consecutive zeros $t_{n}<t_{n+1}<t_{n+2}$, we notice that $y^{\prime \prime}(t)=0$ for some $t \in\left(t_{n+1}, t_{n+2}\right]$. We claim that $t_{n+2}-t_{n} \rightarrow \infty$ as $n \rightarrow \infty$. Otherwise, there exists a subsequence $\left\langle t_{n_{k}}\right\rangle$ of $\left\langle t_{n}\right\rangle$ such that $t_{n_{k}} \rightarrow \infty$ as $k \rightarrow \infty$ and $0<t_{n_{k+2}}-t_{n_{k}} \leq \lambda$ for every $k$, where $\lambda>0$ is a constant. From Corollary 5 we have

$$
\left(t_{n_{k+2}}-t_{n_{k}}\right)^{2}\left[2 q\left(t_{n_{k}}\right)+\int_{t_{n_{k}}}^{t_{n_{k+2}}}\left|q^{\prime}(t)-p(t)\right| d t\right] \geq 4
$$


Then proceeding as above and using (13) we arrive at a contradiction. Thus $t_{n+1}-t_{n} \rightarrow \infty$ or $t_{n+2}-t_{n} \rightarrow \infty$ as $n \rightarrow \infty$.

(ii) Let $0<\sigma \leq 1$. Setting, for $t \geq 0$,

$$
\begin{aligned}
& E_{1}(t)=\left\{s \in\left(t, t+\delta_{0}\right):\left|q^{\prime}(s)-p(s)\right| \leq 1\right\}, \\
& E_{2}(t)=\left\{s \in\left(t, t+\delta_{0}\right):\left|q^{\prime}(s)-p(s)\right|>1\right\},
\end{aligned}
$$

we observe that $E_{1}(t)$ and $E_{2}(t)$ are Lebesgue measurable sets (see [15]) for each $t \geq 0$. Since $E_{1}(t) \cap E_{2}(t)=\emptyset$, we have

$$
\int_{t}^{t+\delta_{0}}\left|q^{\prime}(s)-p(s)\right|^{\sigma} d s=\left(\int_{E_{1}(t)}+\int_{E_{2}(t)}\right)\left|q^{\prime}(s)-p(s)\right|^{\sigma} d s .
$$

Clearly,

$$
m E_{2}(t) \leq \int_{E_{2}(t)}\left|q^{\prime}(s)-p(s)\right|^{\sigma} d s \leq \int_{t}^{t+\delta_{0}}\left|q^{\prime}(s)-p(s)\right|^{\sigma} d s
$$

where $m E$ denotes the Lebesgue measure of a measurable set $E$. Hence $m E_{2}(t) \rightarrow 0$ as $t \rightarrow \infty$ by (12). Since $\left|q^{\prime}(t)-p(t)\right|$ is locally integrable, for every $\varepsilon>0$, there exists a $T_{1}>0$ such that

$$
\int_{E_{2}(t)}\left|q^{\prime}(s)-p(s)\right| d s<\varepsilon / 2
$$

for $t \geq T_{1}$. Further,

$$
\int_{E_{1}(t)}\left|q^{\prime}(s)-p(s)\right| d s \leq \int_{E_{1}(t)}\left|q^{\prime}(s)-p(s)\right|^{\sigma} d s \leq \int_{t}^{t+\delta_{0}}\left|q^{\prime}(s)-p(s)\right|^{\sigma} d s
$$

implies, in view of (12), that

$$
\int_{E_{1}(t)}\left|q^{\prime}(s)-p(s)\right| d s<\varepsilon / 2
$$

for $t \geq T_{2}$, where $T_{2}>0$ is very large. Choosing $T=\max \left\{T_{1}, T_{2}\right\}$, we obtain

$$
\int_{t}^{t+\delta_{0}}\left|q^{\prime}(s)-p(s)\right| d s=\left(\int_{E_{1}(t)}+\int_{E_{2}(t)}\right)\left|q^{\prime}(s)-p(s)\right| d s<\varepsilon / 2+\varepsilon / 2=\varepsilon
$$

for $t \geq T$, that is,

$$
\lim _{t \rightarrow \infty} \int_{t}^{t+\delta_{0}}\left|q^{\prime}(s)-p(s)\right| d s=0
$$

for some $\delta_{0}>0$. Now Theorem 7 shows that $t_{n+1}-t_{n} \rightarrow \infty$ or $t_{n+2}-t_{n} \rightarrow \infty$ as $n \rightarrow \infty$.

Thus the theorem is proved. 
REMARK. If $\sigma>1$, then the local integrability of $|f(t)|^{\sigma}$ implies the local integrability of $|f(t)|$. On the other hand, for $0<\sigma<1,|f(t)|^{\sigma}$ is locally integrable if $|f(t)|$ is locally integrable. These statements can be proved using Hölder's inequality.

Remark. Let $0<\sigma<\infty$. If $f \in L^{\sigma}([0, \infty), \mathbb{R})$, then

$$
\lim _{t \rightarrow \infty} \int_{t}^{t+\delta}|f(s)|^{\sigma} d s=0
$$

for every $\delta>0$. However, the converse is not necessarily true. Let $f(t)=$ $(\log t)^{-1}, t \geq 2$. Then $(\log t)^{2 \sigma}<t$ for large $t$. Hence, for large $T_{0}$,

$$
\int_{T_{0}}^{\infty} \frac{d t}{(\log t)^{\sigma}}>\int_{T_{0}}^{\infty} \frac{d t}{t^{1 / 2}}=\infty
$$

implies that $f \notin L^{\sigma}([2, \infty), \mathbb{R})$. But, for every $\delta>0$,

$$
\lim _{t \rightarrow \infty} \int_{t}^{t+\delta}|f(s)|^{\sigma} d s=0 .
$$

REMARK. In view of the above remark, Theorem 8 is more general than Theorem A. Also the following example confirms this.

ExAmple 1. Consider

$$
y^{\prime \prime \prime}+(\log t)^{-1} y=0, \quad t \geq 2 .
$$

Theorem A cannot be applied to (14) since $(\log t)^{-1} \notin L^{\sigma}([2, \infty), \mathbb{R})$ for $\sigma>1$. On the other hand,

$$
\lim _{t \rightarrow \infty} \int_{t}^{t+\delta}(\log s)^{-\sigma} d s=0
$$

for every $\delta>0$ implies, by Theorem 8 , that $t_{n+1}-t_{n} \rightarrow \infty$ or $t_{n+2}-t_{n} \rightarrow \infty$ as $n \rightarrow \infty$, where $\left\langle t_{n}\right\rangle$ is an increasing sequence of zeros of an oscillatory solution of (14). Since

$$
\int_{2}^{\infty}(\log t)^{-1} d t>\int_{2}^{\sigma} t^{-1} d t=\infty,
$$

from [12] (see Theorem 8) it follows that (14) admits an oscillatory solution.

EXAMPLE 2. A basis of the solution space of the equation

$$
y^{\prime \prime \prime}+t^{-2} y^{\prime}+7 t^{-3} y=0, \quad t \geq 1,
$$

is given by

$$
\left\{t^{-1}, t^{2} \cos (\sqrt{3} \log t), t^{2} \sin (\sqrt{3} \log t)\right\}
$$


The zeros of the oscillatory solution $u(t)=t^{2} \cos (\sqrt{3} \log t)$ of (15) are of the form

$$
t_{n}=\exp \left(\frac{(2 n-1) \pi}{2 \sqrt{3}}\right), \quad n=1,2, \ldots
$$

Clearly, $q(t)=1 / t^{2}>0, q^{\prime}(t)=-2 / t^{3}<0, \lim _{t \rightarrow \infty} q(t)=0$ and

$$
\left|q^{\prime}(t)-p(t)\right|^{\sigma}=9^{\sigma} t^{-3 \sigma}
$$

is locally integrable on $[1, \infty)$ for $0<\sigma<\infty$. Further, for every $\delta>0$,

$$
\int_{t}^{t+\delta}\left|q^{\prime}(s)-p(s)\right|^{\sigma} d s<9^{\sigma} \delta t^{-3 \sigma}
$$

implies that

$$
\lim _{t \rightarrow \infty} \int_{t}^{t+\delta}\left|q^{\prime}(s)-p(s)\right|^{\sigma} d s=0 .
$$

Since $u^{\prime \prime}(t)=-3 \sqrt{3} \sin (\sqrt{3} \log t)-\cos (\sqrt{3} \log t)$, we have

$$
\begin{aligned}
u^{\prime \prime}\left(t_{n}\right) & =-3 \sqrt{3} \sin (n-1 / 2) \pi-\cos (n-1 / 2) \pi, \\
u^{\prime \prime}\left(t_{n+1}\right) & =-3 \sqrt{3} \sin (n+1 / 2) \pi-\cos (n+1 / 2) \pi .
\end{aligned}
$$

Hence $u^{\prime \prime}\left(t_{n}\right) u^{\prime \prime}\left(t_{n+1}\right)<0$ for $n=1,2, \ldots$, because $u^{\prime \prime}\left(t_{n}\right)>0$ or $<0$ according as $n$ is even or odd. Then there exists a $d_{n} \in\left(t_{n}, t_{n+1}\right)$ such that $u^{\prime \prime}\left(d_{n}\right)=0$. From Theorem 8 it follows that $t_{n+1}-t_{n} \rightarrow \infty$ as $n \rightarrow \infty$. Indeed,

$$
t_{n+1}-t_{n}=\left(e^{\pi / \sqrt{3}}-1\right) \exp \left(\frac{(2 n-1) \pi}{2 \sqrt{3}}\right) \rightarrow \infty \quad \text { as } n \rightarrow \infty .
$$

As we are in Case I, (10) yields

$$
\left(t_{2}-t_{1}\right)^{2}\left[2 q\left(t_{1}\right)+\int_{t_{1}}^{t_{2}}\left|q^{\prime}(t)-p(t)\right| d t\right] \geq 4 .
$$

Indeed,

$$
\begin{aligned}
\left(t_{2}-t_{1}\right)^{2}\left[2 q\left(t_{1}\right)\right. & \left.+\int_{t_{1}}^{t_{2}}\left|q^{\prime}(t)-p(t)\right| d t\right] \\
& =e^{\pi / \sqrt{3}}\left(e^{\pi / \sqrt{3}}-1\right)^{2}\left[2 e^{-\pi / \sqrt{3}}+\frac{9}{2}\left(e^{-\pi / \sqrt{3}}-e^{-\sqrt{3} \pi}\right)\right] \\
& =3547.4982[5.4695653-5.4608985] \\
& =30.745457>4 .
\end{aligned}
$$

Thus, this example illustrates Corollary 4 and Theorem 8 . 
Remark. In Example 2, it is not easy to determine $d_{n}$ explicitly. Hence it is not possible to verify (5). We can do this in the following example.

Example 3. Consider

$$
y^{\prime \prime \prime}-(\sin t) y^{\prime}+(\cos t) y=0, \quad t \geq 0 .
$$

Clearly, $y(t)=1+\sin t$ is an oscillatory solution of the equation with $y(3 \pi / 2)=0, y(7 \pi / 2)=0, y(t)>0$ for $t \in(3 \pi / 2,7 \pi / 2), y^{\prime}(3 \pi / 2)=0$, $y^{\prime}(5 \pi / 2)=0, y^{\prime}(7 \pi / 2)=0, y^{\prime \prime}(2 \pi)=0$ and $y^{\prime \prime}(3 \pi)=0$. Here $q(t)=-\sin t$ and $p(t)=\cos t$. Since $|q(2 \pi)|=0=|q(3 \pi)|$, we may take $d=2 \pi$ or $3 \pi$. Let $d=2 \pi$. Then

$$
\begin{aligned}
\int_{a}^{b}|q(t)| d t & =\int_{3 \pi / 2}^{7 \pi / 2}|\sin t| d t \\
& =\int_{3 \pi / 2}^{2 \pi}(-\sin t) d t+\int_{2 \pi}^{3 \pi} \sin t d t+\int_{3 \pi}^{7 \pi / 2}(-\sin t) d t=4
\end{aligned}
$$

and

$$
\int_{a}^{b}\left|q^{\prime}(t)-p(t)\right| d t=2 \int_{3 \pi / 2}^{7 \pi / 2}|\cos t| d t=2 \int_{3 \pi / 2}^{5 \pi / 2} \cos t d t-2 \int_{5 \pi / 2}^{7 \pi / 2} \cos t d t=8 .
$$

Hence

$$
\begin{aligned}
(b-a)\left[\int_{a}^{b}|q(t)| d t+(b-a)|q(d)|+\right. & \left.(b-a) \int_{a}^{b}\left|q^{\prime}(t)-p(t)\right| d t\right] \\
& =2 \pi[4+16 \pi]=8 \pi+32 \pi^{2}>4 .
\end{aligned}
$$

REMARK. Example 3 indicates that the inequalities (5) and (6) could be improved substantially.

3. The following two Opial-type inequalities (see [1]) are needed for our work in this section:

THEOREM 9. If $f$ is absolutely continuous on $[a, b]$ with $f(a)=0$ and $g \in L^{2}([a, b], \mathbb{R})$, then

$$
\int_{a}^{b} g(t)|f(t)| \cdot\left|f^{\prime}(t)\right| d t \leq k \int_{a}^{b}\left|f^{\prime}(t)\right|^{2} d t
$$

where

$$
k=\frac{1}{\sqrt{2}}\left(\int_{a}^{b} g^{2}(t)(t-a) d t\right)^{1 / 2}
$$

with equality if and only if $f \equiv 0$ or $f$ is linear and $g$ is constant. 
If $f(a)=0$ in Theorem 9 is replaced by $f(b)=0$, then (17) holds with $k$ given by

$$
k=\frac{1}{\sqrt{2}}\left(\int_{a}^{b} g^{2}(t)(b-t) d t\right)^{1 / 2} .
$$

THEOREM 10. If $f$ is absolutely continuous on $[a, b]$ with $f(a)=0$ or $f(b)=0$ and $1 \leq \sigma \leq 2$, then

$$
\int_{a}^{b}|f(t)|^{\sigma}\left|f^{\prime}(t)\right|^{\sigma} d t \leq T(\sigma)(b-a) \int_{a}^{b}\left|f^{\prime}(t)\right|^{2} d t
$$

where

$$
T(\sigma)= \begin{cases}1 / 2, & \sigma=1, \\ 4 / \pi^{2}, & \sigma=2, \\ \frac{2-\sigma}{2 \sigma}\left(\frac{1}{\sigma}\right)^{2 \sigma-2} I^{-\sigma}, & 1<\sigma<2,\end{cases}
$$

with

$$
I=\int_{0}^{1}\left[1+\frac{2(\sigma-1)}{2-\sigma} t\right]^{-2}[1+(\sigma-1) t]^{(1 / \sigma)-1} d t .
$$

For $\sigma=1$, equality holds in (20) only for $f$ linear.

We consider

$$
y^{\prime \prime \prime}+q(t) y^{\prime}+p(t) y=0,
$$

where $p, q \in C([a, b], \mathbb{R})$ and $0 \leq a<b$.

THEOREM 11. Suppose that $y(t)$ is a solution of $\left(4^{\prime}\right)$ with $y(a)=0$, $y^{\prime}(a)=0, y^{\prime}(c)=0(a<c<b)$ and $y(t)$ has no extreme value in $(a, c)$. Then

$$
8(d-a)^{2} \int_{a}^{d}\left[P(t)+\frac{1}{2} q(t)\right]^{2}(t-a) d t \geq 1,
$$

where $y^{\prime \prime}(d)=0, a<d<c$, and $P(t)=\int_{t}^{d} p(s) d s$.

If $y^{\prime}(c)=0, y(b)=0=y^{\prime}(b)$ and $y(t)$ has no extreme value in $(c, b)$, then

$$
8\left(b-d^{\prime}\right)^{2} \int_{d^{\prime}}^{b}\left[P(t)+\frac{1}{2} q(t)\right]^{2}(b-t) d t \geq 1,
$$

where $y^{\prime \prime}\left(d^{\prime}\right)=0, c<d^{\prime}<b$, and $P(t)=\int_{d^{\prime}}^{t} p(s) d s$. 
Proof. Consider the case $y(a)=0=y^{\prime}(a)$ and $y^{\prime}(c)=0$. Integrating

$$
y(t) y^{\prime \prime \prime}(t)+q(t) y(t) y^{\prime}(t)+p(t) y^{2}(t)=0
$$

from $a$ to $d$ we obtain

$$
\int_{a}^{d} y^{\prime}(t) y^{\prime \prime}(t) d t=\int_{a}^{d} q(t) y(t) y^{\prime}(t) d t+\int_{a}^{d} p(t) y^{2}(t) d t .
$$

Hence

$$
\begin{aligned}
\frac{1}{2}\left(y^{\prime}(d)\right)^{2} & =\int_{a}^{d} q(t) y(t) y^{\prime}(t) d t-\int_{a}^{d} P^{\prime}(t) y^{2}(t) d t \\
& =\int_{a}^{d} q(t) y(t) y^{\prime}(t) d t+2 \int_{a}^{d} P(t) y(t) y^{\prime}(t) d t .
\end{aligned}
$$

Thus, by Theorem 9 ,

$$
\begin{aligned}
\frac{1}{4}\left(y^{\prime}(d)\right)^{2} & =\int_{a}^{d}\left[P(t)+\frac{1}{2} q(t)\right] y(t) y^{\prime}(t) d t \\
& \leq \int_{a}^{d}\left|P(t)+\frac{1}{2} q(t)\right| \cdot|y(t)| \cdot\left|y^{\prime}(t)\right| d t \\
& \leq \frac{1}{\sqrt{2}}\left[\int_{a}^{d}\left(P(t)+\frac{1}{2} q(t)\right)^{2}(t-a) d t\right]^{1 / 2} \int_{a}^{d}\left(y^{\prime}(t)\right)^{2} d t \\
& \leq \frac{\left(y^{\prime}(d)\right)^{2}(d-a)}{\sqrt{2}}\left[\int_{a}^{d}\left(P(t)+\frac{1}{2} q(t)\right)^{2}(t-a) d t\right]^{1 / 2} .
\end{aligned}
$$

Dividing by $\left(y^{\prime}(d)\right)^{2}$ and then squaring yields the required inequality. Proceeding as above we can get the other inequality when $y^{\prime}(c)=0$ and $y(b)=0=y^{\prime}(b)$. This completes the proof of the theorem.

REMARK. If $q(t) \equiv 0$, then (22) and (23) take, respectively, the forms

$$
2(d-a)^{2} \max _{a \leq t \leq d}\left|\int_{t}^{d} p(s) d s\right| \geq 1
$$

and

$$
2\left(b-d^{\prime}\right)^{2} \max _{d^{\prime} \leq t \leq b}\left|\int_{d^{\prime}}^{t} p(s) d s\right| \geq 1 .
$$

REMARK. The inequalities (22) and (23) fail to provide us the lower bound of the spacing $c-a$ or $b-c$. In the following we derive some inequalities for this purpose. 
THEOREM 12. Let $q$ be once continuously differentiable. If $y(t)$ is a solution of $\left(4^{\prime}\right)$ with $y(a)=0, y^{\prime}(a)=0, y^{\prime}(c)=0(a<c<b)$ and $y(t)$ has no extreme value in $(a, c)$, then

$$
(c-a)\left[\int_{a}^{c}|q(t)| d t+|q(d)|(c-a)+(c-a) \int_{a}^{c}\left|q^{\prime}(t)-p(t)\right| d t\right] \geq 1,
$$

where $y^{\prime \prime}(d)=0, a<d<c$. If $y^{\prime}(c)=0, y(b)=0, y^{\prime}(b)=0$ and $y(t)$ has no extreme value in $(c, b)$, then

$$
(b-c)\left[\int_{c}^{b} q(t) d t+q\left(d^{\prime}\right)(b-c)+(b-c) \int_{c}^{b}\left|q^{\prime}(t)-p(t)\right| d t\right] \geq 1,
$$

where $y^{\prime \prime}\left(d^{\prime}\right)=0, c<d^{\prime}<b$.

Proof. There exists $d \in(a, c)$ such that $y^{\prime \prime}(d)=0$. Setting

$$
M=\max \{|y(t)|: t \in[a, c]\}=|y(c)|,
$$

we may write

$$
M=|y(c)|=\left|\int_{a}^{c} y^{\prime}(t) d t\right| \leq \int_{a}^{c}\left|y^{\prime}(t)\right| d t
$$

Hence using the Cauchy-Schwarz inequality first and then integrating by parts we get

$$
\begin{aligned}
M^{2} & \leq\left[\int_{a}^{c}\left|y^{\prime}(t)\right| d t\right]^{2} \leq(c-a) \int_{a}^{c}\left(y^{\prime}(t)\right)^{2} d t \\
& =-(c-a) \int_{a}^{c} y(t) y^{\prime \prime}(t) d t \leq(c-a) \int_{a}^{c}|y(t)| \cdot\left|y^{\prime \prime}(t)\right| d t .
\end{aligned}
$$

Proceeding as in the proof of Theorem 2 we obtain

$$
\left|y^{\prime \prime}(t)\right| \leq M\left[|q(t)|+|q(d)|+\int_{a}^{c}\left|q^{\prime}(t)-p(t)\right| d t\right] .
$$

Hence

$$
1 \leq(c-a)\left[\int_{a}^{c}|q(t)| d t+(c-a)|q(d)|+(c-a) \int_{a}^{c}\left|q^{\prime}(t)-p(t)\right| d t\right] .
$$

Similarly, setting $M^{\prime}=\max \{|y(t)|: t \in[c, b]\}=|y(c)|$ and observing that there exists a $d^{\prime} \in(c, b)$ such that $y^{\prime \prime}\left(d^{\prime}\right)=0$, we obtain the other inequality. Thus the theorem is proved.

REMARK. It is not always easy to locate the point $d$ or $d^{\prime}$ where $y^{\prime \prime}$ vanishes. 
TheOREM 13. Let $y(t)$ be a solution of $\left(4^{\prime}\right)$ with $y(a)=0, y^{\prime}(a)=0$, $y^{\prime}(c)=0(a<c<b)$ and let $y(t)$ have no extreme value in $(a, c)$. Then

$$
4 T^{1 / \sigma}(\sigma)(d-a)^{(\sigma+1) / \sigma}\left[\int_{a}^{d}\left|P(t)+\frac{1}{2} q(t)\right|^{\sigma^{\prime}} d t\right]^{1 / \sigma^{\prime}} \geq 1,
$$

where $1 \leq \sigma \leq 2,1 / \sigma+1 / \sigma^{\prime}=1, T(\sigma)$ is given by $(21)$ and $y^{\prime \prime}(d)=0$ $(a<d<c)$. If $\sigma=1$, then

$$
\left[\int_{a}^{d}\left|P(t)+\frac{1}{2} q(t)\right|^{\sigma^{\prime}} d t\right]^{1 / \sigma^{\prime}}=\max \left\{\left|P(t)+\frac{1}{2} q(t)\right|: a \leq t \leq d\right\} .
$$

If $y(t)$ is a solution of $\left(4^{\prime}\right)$ with $y^{\prime}(c)=0, y(b)=0, y^{\prime}(b)=0(a<c<b)$ and $y(t)$ has no extreme value in $(c, b)$, then

$$
4 T^{1 / \sigma}(\sigma)\left(b-d^{\prime}\right)^{(\sigma+1) / \sigma}\left[\int_{d^{\prime}}^{b}\left|P(t)+\frac{1}{2} q(t)\right|^{\sigma^{\prime}} d t\right]^{1 / \sigma^{\prime}} \geq 1,
$$

where $y^{\prime \prime}\left(d^{\prime}\right)=0\left(c<d^{\prime}<b\right)$. If $\sigma=1$, then

$$
\left[\int_{d^{\prime}}^{b}\left|P(t)+\frac{1}{2} q(t)\right|^{\sigma^{\prime}} d t\right]^{1 / \sigma^{\prime}}=\max \left\{\left|P(t)+\frac{1}{2} q(t)\right|: d^{\prime} \leq t \leq b\right\} .
$$

Proof. Consider the case $y(a)=0, y^{\prime}(a)=0$ and $y^{\prime}(c)=0$. Proceeding as in the proof of Theorem 11 and using Hölder's inequality and Theorem 10, we obtain

$$
\begin{aligned}
\frac{1}{4}\left(y^{\prime}(d)\right)^{2} & \leq \int_{a}^{d}\left|P(t)+\frac{1}{2} q(t)\right| \cdot|y(t)| \cdot\left|y^{\prime}(t)\right| d t \\
& \leq\left[\int_{a}^{d}\left|P(t)+\frac{1}{2} q(t)\right|^{\sigma^{\prime}} d t\right]^{1 / \sigma^{\prime}}\left[\int_{a}^{d}\left(|y(t)| \cdot\left|y^{\prime}(t)\right|\right)^{\sigma} d t\right]^{1 / \sigma} \\
& \leq T^{1 / \sigma}(\sigma)(d-a)^{1 / \sigma}\left[\int_{a}^{d}\left|P(t)+\frac{1}{2} q(t)\right|^{\sigma^{\prime}} d t\right]^{1 / \sigma^{\prime}}\left[\int_{a}^{d}\left(y^{\prime}(t)\right)^{2} d t\right] \\
& \leq T^{1 / \sigma}(\sigma)(d-a)^{(\sigma+1) / \sigma}\left(y^{\prime}(d)\right)^{2}\left[\int_{a}^{d}\left|P(t)+\frac{1}{2} q(t)\right|^{\sigma^{\prime}} d t\right]^{1 / \sigma^{\prime}} .
\end{aligned}
$$

Cancelling $\left(y^{\prime}(d)\right)^{2}$ yields the required inequality. Similarly, the other inequality is obtained by considering $y^{\prime}(c)=0, y(b)=0=y^{\prime}(b)$. In this case, there exists a $d^{\prime} \in(c, b)$ such that $y^{\prime \prime}\left(d^{\prime}\right)=0$. Thus the theorem is proved.

EXAmple 4. Consider (16) with its solution $y(t)=1+\sin t, t \geq 0$. We have $y(3 \pi / 2)=0, y^{\prime}(3 \pi / 2)=0, y^{\prime}(5 \pi / 2)=0$ and $y^{\prime \prime}(2 \pi)=0$. Thus $a=3 \pi / 2, c=5 \pi / 2$ and $d=2 \pi$. Clearly, $y(t)$ has no extreme value in 
$(3 \pi / 2,5 \pi / 2)$. Since

$$
P(t)=\int_{t}^{d} p(\theta) d \theta=\int_{t}^{2 \pi} \cos \theta d \theta=-\sin t
$$

we have

$$
\begin{aligned}
\int_{a}^{d}\left[P(t)+\frac{1}{2} q(t)\right]^{2}(t-a) d t & =\frac{9}{4} \int_{3 \pi / 2}^{2 \pi}\left(t-\frac{3 \pi}{2}\right) \sin ^{2} t d t \\
& =\frac{9}{8} \int_{3 \pi / 2}^{2 \pi} t(1-\cos t) d t-\frac{27 \pi}{16} \int_{3 \pi / 2}^{2 \pi}(1-\cos t) d t \\
& =\frac{9}{8}\left(\frac{7 \pi^{2}}{8}-\frac{1}{2}\right)-\frac{27 \pi^{2}}{32}=\frac{9}{16}\left[\frac{\pi^{2}}{4}-1\right]
\end{aligned}
$$

and hence

$$
8(d-a)^{2} \int_{a}^{d}\left[P(t)+\frac{1}{2} q(t)\right]^{2}(t-a) d t=\frac{9 \pi^{2}}{8}\left[\frac{\pi^{2}}{4}-1\right]>1 .
$$

Similarly, the inequality (23) may be verified taking $c=5 \pi / 2, d^{\prime}=3 \pi$ and $b=7 \pi / 2$.

4. Discussion. (i) If $y(t)$ is a solution of

$$
y^{\prime \prime \prime}+q(t) y^{\prime}+p(t) y=f(t)
$$

where $p$ and $q$ are as in (4) and $f$ is a real-valued locally integrable function on $[0, \infty)$, with $y(a)=0=y(b)(0 \leq a<b)$ and $y(t) \neq 0$ for $t \in(a, b)$, then proceeding as in the proof of Theorem 2 we may obtain

$$
\begin{aligned}
(b-a)\left[\int_{a}^{b}|q(t)| d t+(b-a)|q(d)|+(b-a) \int_{a}^{b} \mid\right. & q^{\prime}(t)-p(t) \mid d t \\
& \left.+\frac{b-a}{M} \int_{a}^{b}|f(t)| d t\right] \geq 4
\end{aligned}
$$

provided that there exists a $d \in[a, b]$ such that $y^{\prime \prime}(d)=0$, where $M=$ $\max \{|y(t)|: t \in[a, b]\}$. If $y^{\prime \prime}(t) \neq 0$ for $t \in[a, b]$, then we consider three consecutive zeros $a, b$ and $a^{\prime}\left(0 \leq a<b<a^{\prime}\right)$ of $y(t)$ to obtain

$$
\begin{array}{r}
\left(a^{\prime}-a\right)\left[\int_{a}^{a^{\prime}}|q(t)| d t+\left(a^{\prime}-a\right)|q(d)|+\left(a^{\prime}-a\right) \int_{a}^{a^{\prime}}\left|q^{\prime}(t)-p(t)\right| d t\right. \\
\left.+\frac{a^{\prime}-a}{M^{\prime}} \int_{a}^{a^{\prime}}|f(t)| d t\right] \geq 4,
\end{array}
$$


where $M^{\prime}=\max \left\{|y(t)|: t \in\left[a, a^{\prime}\right]\right\}$ and $d \in\left[b, a^{\prime}\right]$ is such that $y^{\prime \prime}(d)=0$. If $0 \leq a^{\prime} \leq a<b$, then the above inequality changes accordingly. In the following, we consider an example where the second situation indeed occurs.

EXAMPLE 5. We may observe that $y(t)=5 t^{2}-t^{4}-4 \equiv\left(t^{2}-1\right)\left(4-t^{2}\right)$ is a solution of

$$
y^{\prime \prime \prime}+4 y^{\prime}+4 t y=4 t^{3}\left(t^{2}-1\right), \quad t \geq-2,
$$

with $y(-1)=0, y(1)=0, y(2)=0, y^{\prime}(0)=0$ and $y^{\prime}(\sqrt{5 / 2})=0$. It is interesting to note that $y^{\prime \prime}(t) \neq 0$ for $t \in[1,2]$. However, $y^{\prime \prime}( \pm \sqrt{5 / 6})=0$ where $\pm \sqrt{5 / 6} \in(-1,1)$.

It would be interesting to obtain a homogeneous equation where this situation occurs.

(ii) Some authors (see $[3,7,10]$ and the references therein) have succeeded in obtaining an upper bound of $(b-a) \int_{a}^{b}|p(t)| d t$ and improved its lower bound concerning (1). Yet no such attempt has been made for (3). It would be interesting to obtain upper bounds of the inequalities (8) and (9) concerning (3) and of the inequalities (5) and (6) concerning (4). It has already been remarked that the lower bounds of these inequalities could be improved.

(iii) If $\left\langle t_{n}\right\rangle$ is a sequence of zeros of $1+\sin t$, then $\left\langle t_{n+1}-t_{n}\right\rangle$ is bounded. It would be interesting to obtain sufficient conditions on $p$ and $q$ so that $\left\langle t_{n+1}-t_{n}\right\rangle$ or $\left\langle t_{n+2}-t_{n}\right\rangle$ is bounded, where $\left\langle t_{n}\right\rangle$ is a sequence of zeros of an oscillatory solution of (4).

\section{References}

[1] R. C. Brown and D. B. Hinton, Opial's inequality and oscillation of 2nd order equations, Proc. Amer. Math. Soc. 125 (1997), 1123-1129.

[2] R. S. Dahiya and B. Singh, A Liapunov inequality and nonoscillation theorem for a second order nonlinear differential-difference equation, J. Math. Phys. Sci. 7 (1973), 163-170.

[3] S. B. Eliason, The integral $T \int_{-T / 2}^{T / 2} p(t) d t$ and the boundary value problem $x^{\prime \prime}+$ $p(t) x=0, x(-T / 2)=x(T / 2)=0$, J. Differential Equations 4 (1968), 646-660.

[4] -, A Liapunov inequality for a certain second order nonlinear differential equation, J. London Math. Soc. 2 (1970), 461-466.

[5] - Liapunov type inequalities for certain second order functional differential equations, SIAM J. Appl. Math. 27 (1974), 180-199.

[6] -, Distance between zeros of certain differential equations having delayed arguments, Ann. Mat. Pura Appl. 106 (1975), 273-291.

[7] A. M. Fink, Comparison theorems for $\int_{a}^{b} p$ with $p$ an admissible sub or superfunction, J. Differential Equations 5 (1969), 49-54.

[8] P. Hartman, Ordinary Differential Equations, Wiley, New York, 1964. 
[9] A. M. Liapunov, Problème général de la stabilité du mouvement, Ann. of Math. Stud. 17, Princeton Univ. Press, Princeton, NJ, 1949.

[10] D. F. St. Mary and S. B. Eliason, Upper bounds of $T \int_{-T / 2}^{T / 2} p(t) d t$ and the differential equation $x^{\prime \prime}+p(t) x=0$, J. Differential Equations 6 (1969), 154-160.

[11] B. G. Pachpatte, On Liapunov-type inequalities for certain higher order differential equations, J. Math. Anal. Appl. 195 (1995), 527-536.

[12] N. Parhi and P. Das, On asymptotic properties of solutions of a class of third order differential equations, Proc. Amer. Math. Soc. 110 (1990), 387-393.

[13] N. Parhi and S. Panigrahi, On Liapunov-type inequality for third-order differential equations, J. Math. Anal. Appl. 233 (1999), 445-460.

[14] W. T. Patula, On the distance between zeros, Proc. Amer. Math. Soc. 52 (1975), 247-251.

[15] H. L. Royden, Real Analysis, Macmillan, New York, 1968.

[16] J. Yan, On the distance between zeros and the limit-point problem, Proc. Amer. Math. Soc. 107 (1989), 971-975.

Department of Mathematics

Berhampur University

Berhampur 760007, India 